\title{
Interval Estimation Naïve Bayes
}

\author{
V. Robles ${ }^{1}$, P. Larrañaga ${ }^{2}$, J.M. Peña ${ }^{1}$, E. Menasalvas ${ }^{1}$, M.S. Pérez ${ }^{1}$ \\ 1 Department of Computer Architecture and Technology, Technical University of Madrid, \\ Madrid, Spain, \{vrobles, jmpena, emenasalvas, mperez\}@ fi.upm.es \\ 2 Department of Computer Science and Artificial Intelligence, University of the Basque \\ Country, San Sebastián, Spain, ccplamup@si.ehu.es
}

\begin{abstract}
Recent work in supervised learning has shown that a surprisingly simple Bayesian classifier with assumptions of conditional independence among features given the class, called naïve Bayes, is competitive with state of the art classifiers. On this paper a new naive Bayes classifier called Interval Estimation naïve Bayes is proposed. Interval Estimation naïve Bayes performs on two phases. On the first phase an interval estimation of each probability necessary to specify the naïve Bayes is estimated. On the second phase the best combination of values inside these intervals is calculated with a heuristic search that is guided by the accuracy of the classifiers. The founded values in the search are the new parameters for the naïve Bayes classifier. Our new approach has shown to be quite competitive related to simple naïve Bayes. Experimental tests have been done with 21 data sets from the UCI repository.
\end{abstract}

\section{Introduction}

The naïve Bayes classifier $[3,8]$ is a probabilistic method for classification. It can be used to determine the probability that an example belongs to a class given the values of attributes. The naïve Bayes classifier guarantees optimal induction given a set of explicit assumptions [1]. However, it is known that some of these assumptions are not compliant in many induction scenarios, for instance, the condition of variable independence respecting to the class variable. Improvements of accuracy has been demonstrated by a number of approaches, collectively named semi naïve Bayes classifiers, which try to adjust the naïve Bayes to deal with a-priori unattended assumptions.

Previous semi naïve Bayes classifiers can be divided into three groups, depending on different pre-/post-processing issues: (i) to manipulate the variables to be employed prior to application of naïve Bayes induction $[12,14,18]$, (ii) to select subsets of the training examples prior to the application of naïve Bayes classification $[10,13]$ and (iii) to correct the probabilities produced by the standard naïve Bayes [21,24].

We propose a new semi naïve Bayes approach named Interval Estimation naïve Bayes (IENB), that tries to break the assumption of independence of the variables given the class by searching for the best combination of naïve Bayes probabilities inside its confidence intervals. There are some related work on this approximation. In [6] it is described an algorithm named Iterative Bayes, that tries to improve the conditional probabilities of naïve Bayes in an iterative way with a hill-climbing approach. In [19] is presented an algorithm named Robust Bayes Classifier $(R B C)$, a naïve Bayes classifier 
designed for the case in which learning instances are incomplete. RBC takes into account all the possibilities for the missing values, calculating an interval for each naïve Bayes probability. In $[22,23]$ is presented a naïve Bayes extension based on credal approximation naïve Credal Classifier (NCC). Credal sets represent probability distributions as points that belong to a close and fenced geometrical regions.

Interval Estimation naïve Bayes has been implemented in Visual $\mathrm{C}++$ and the experimental evaluation has been done with 21 problems from the UCI database [17].

The outline of this paper is as follows: Section 2 presents the naïve Bayes classifier. Section 3 is a brief introduction to statistical inference. Section 4 presents the new algorithm Interval Estimation naïve Bayes. Section 5 illustrates the results with the UCI experiments. Section 6 gives the conclusions and suggests further future work.

\section{Naïve Bayes}

The naïve Bayes classifier $[3,8]$ is a probabilistic method for classification. It performs and approximate calculation of the probability that an example belongs to a class given the values of variables. The simple naïve Bayes classifier is one of the most successful algorithms on many classification domains. In spite of its simplicity, it is shown to be competitive with other more complex approaches in several specific domains.

This classifier learns from training data the conditional probability of each variable $X_{k}$ given the class label $c$. Classification is then done by applying Bayes rule to compute the probability of $C$ given the particular instance of $X_{1}, \ldots, X_{n}$,

$$
P\left(C=c \mid X_{1}=x_{1}, \ldots, X_{n}=x_{n}\right)
$$

Naïve Bayes is founded on the assumption than variables are independent. Therefore this probability (given the value of the class) are formuled as follows,

$$
P\left(C=c \mid X_{1}=x_{1}, \ldots, X_{n}=x_{n}\right) \propto P(C=c) \prod_{k=1}^{n} P\left(X_{k}=x_{k} \mid C=c\right)
$$

This equation is highly appropriate for learning from data, since the probabilities $p_{i}=P\left(C=c^{i}\right)$ and $p_{k, r}^{i}=P\left(X_{k}=x_{k}^{r} \mid C=c^{i}\right)$ may be estimated from training data. The result of the classification is the class with highest probability.

In naïve Bayes these parameters are estimated using a point estimation (see section $\mathrm{xxx})$. The first step in the new algorithm we propose is based on an interval estimation (see section $\mathrm{xxx}$ ).

\section{Parameter estimation}

Statistical inference studies a collection of data based on a sample of these ones. This sample represents the part of population considered in the analysis. Amongst other things, statistical inference studies the problem known as "estimation problem".

There are two ways of accomplishing this task: 
- Point Estimation: Point estimation uses a sample with the aim of assigning a single value to a parameter. Maximum likelihood method and method of moments are used in this context.

- Interval Estimation: This technique calculates for each sample an interval that probably contains the parameter. This interval is called confidence interval. The probability of a parameter to be included in an interval is known as confidence level.

\subsection{Point estimation of parameters in naïve Bayes}

Considering the instances of the database $\mathcal{D}=\left\{\left(x_{1}, c_{1}\right), \ldots,\left(x_{N}, c_{N}\right)\right\}$ as a random sample on size $N$ where the predictor variables $X_{1}, \ldots, X_{N}$ follow Bernoulli distributions and using the maximum likelihood method, the next intuitive results are reached:

$$
\hat{P}_{k, r}^{i}=\frac{N_{k r i}}{N_{i}}
$$

where,

$N_{k r i}$ denotes the number of instances where $X_{k}=x_{k}^{r}$ and $C=c^{i}$

$N_{i}$ denotes the number of instances where $C=c^{i}$

$$
\hat{P}_{i}=\frac{N_{i}}{N}
$$

being $N$ is the size of the database.

\subsection{Interval estimation of parameters in IENB}

In the case of IENB what we need is to calculate the confidence intervals of the parameters. This estimation is achieved by the calculation of the sum of the variables in the sample, which generate a binomial distribution that can be approximated by a normal distribution, given the next result,

$$
p \in\left(\hat{p}-z_{\alpha} \sqrt{\frac{\hat{p}(1-\hat{p})}{M}} ; \hat{p}+z_{\alpha} \sqrt{\frac{\hat{p}(1-\hat{p})}{M}}\right)
$$

where,

$\hat{p}$ is the point estimation of the probability

$z_{\alpha}$ is the $\left(1-\frac{\alpha}{2}\right)$ percentil in the $\mathcal{N}(0,1)$

$M$ is the corresponding sample size $\left(N_{i}\right.$ or $\left.N\right)$

\section{Interval Estimation Naïve Bayes - IENB}

We propose a new semi naïve Bayes approach named interval estimation naïve Bayes (IENB). In this approach, instead of calculating the point estimation of the conditional probabilities from data, as simple naïve Bayes does, confidence intervals are calculated. After that, by searching for the best combination of values into these intervals, we seek to break the assumption of independence among variables the simple naïve Bayes does. 
This search is carry out by a heuristic search algorithm and is guided by the accuracy of the classifiers.

As it is pictured in figure 2 at the end of the paper, there are three main important aspects in IENB algorithm:

\section{- Calculation of confidence intervals}

Given the dataset, the first step is to calculate the confidence intervals for each conditional probability and for each class probability. For the calculation of the intervals it must be computed first the point estimations of these same parameters (see section 3.1).

In this way, each conditional probability that has to be estimated from the dataset $\hat{p}_{k, r}^{i}=\hat{P}\left(X_{k}=x_{k}^{r} \mid C=c^{i}\right)$ must be computed with the next confidence interval, as it is introduced in section 3.1. The contribution of this paper is addressed towards this approach.

For $k=1, \ldots, n ; i=1, \ldots, r_{0} ; r=1, \ldots, r_{k}$

$$
\left(\hat{p}_{k, r}^{i}-z_{\alpha} \sqrt{\frac{\hat{p}_{k, r}^{i}\left(1-\hat{p}_{k, r}^{i}\right)}{N_{i}}} ; \hat{p}_{k, r}^{i}+z_{\alpha} \sqrt{\frac{\hat{p}_{k, r}^{i}\left(1-\hat{p}_{k, r}^{i}\right)}{N_{i}}}\right)
$$

where,

$\mathrm{r}$ are the possible values of variable $X_{k}$

$i$ are the possible values of the class

$\hat{p}_{k, r}^{i}$ is the point estimation of the conditional probability $P\left(X_{k}=x_{k}^{r} \mid C=c^{i}\right)$

$z_{\alpha}$ is the $\left(1-\frac{\alpha}{2}\right)$ percentil in the $\mathcal{N}(0,1)$

$N_{i}$ denotes the number of instances where $C=c^{i}$

Also, in a similar way, the probabilities for the class values $\hat{p}_{i}=\hat{P}\left(C=c^{i}\right)$ are estimated with the next confidence interval,

$$
\left(\hat{p}_{i}-z_{\alpha} \sqrt{\frac{\hat{p}_{i}\left(1-\hat{p}_{i}\right)}{N}} ; \hat{p}_{i}+z_{\alpha} \sqrt{\frac{\hat{p}_{i}\left(1-\hat{p}_{i}\right)}{N}}\right)
$$

where,

$\hat{p}_{i}^{i}$ is the point estimation of the probability $P\left(C=c_{i}\right)$

$z_{\alpha}$ is the $\left(1-\frac{\alpha}{2}\right)$ percentil in the $\mathcal{N}(0,1)$

$N$ is the number of cases in dataset

\section{- Search space definition}

Once the confidence intervals are estimated from the dataset, it is possible to generate as many naïve Bayes classifiers as we want. The parameters of these naïve Bayes classifiers must only be taken inside theirs corresponding confidence interval.

In this way, each naïve Bayes classifier is going to be represented with the next tupla of dimension $r_{0}\left(1+\sum_{i=1}^{n} r_{i}\right)$

$$
\left(p_{1}^{*}, \ldots, p_{r_{0}}^{*}, p_{1,1}^{* 1}, \ldots, p_{1,1}^{* r_{0}}, \ldots, p_{1, r_{1}}^{* r_{0}}, \ldots, p_{n, r_{n}}^{* r_{0}}\right)
$$


where,

$p^{*}$ are the selected values inside each confidence interval.

Thus, the search space for the heuristic optimization algorithm is composed of all the valid tuplas. A tupla is valid when it represents a valid naïve Bayes classifier. Formally,

$$
\sum_{i=1}^{r_{0}} p_{i}^{*}=1 ; \forall k \forall i \sum_{r=1}^{r_{k}} p_{k, r}^{* i}=1
$$

Finally, each generated individual must be evaluated with a fitness function. This fitness function it is based on the percentage of successful predictions on each dataset, what means that we are carrying out one wrapper approach.

\section{- Heuristic search for the best individual}

Once the individuals and the search space are defined, one heuristic optimization algorithm is ran in order to find the best individual.

To deal with the heuristic search in this work, EDAs -estimation of distribution algorithms- algorithms have been selected. EDAs $[16,15]$ are non-deterministic, stochastic and heuristic search strategies that belong to the evolutionary computation approaches. In EDAs, the number of solutions or individuals are created every generation, evolving once and again until a satisfactory solution is achieved. In brief, the characteristic that most differentiates EDAs from other evolutionary search strategies such as GAs is that the evolution from a generation to the next one is done by estimating the probability distribution of the fittest individuals, and afterwards by sampling the induced model. This avoids the use of crossing or mutation operators, and, therefore, the number of parameters that EDAs requires is reduced considerably.

\section{Experimentation}

\subsection{Datasets}

Results are compared for 21 classical datasets -see table 1-, also used by other authos [5]. All the datsets belong to the UCI repository [17], with the exception of $m-o n-f-3$ 7-10 and corral. These two artificial data sets were designed to evaluate methods for feature subset selection [9].

\subsection{Experimental methodology}

To estimate the prediction accuracy for each classifier our own implementation of a naïve Bayes classifier has been programmed. This implementation uses the Laplace correction for the point estimation of the conditional probabilities [7,9] and deals with missing values as recommended in [1].

However, our new algorithm does not handle continuous attributes. Thus, a discretization step with the method recommended in [2] has been performed usign MLC++ 
Table 1. Description of the data sets used in the experiments

\begin{tabular}{|c|c|c|c|c|c|c|}
\hline Name & \multicolumn{3}{|c|}{ Attributes } & \multicolumn{3}{|c|}{ Instances } \\
\hline breast & 10 & 10 & 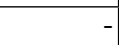 & \begin{tabular}{|l|}
2 \\
\end{tabular} & \begin{tabular}{|l}
699 \\
\end{tabular} & \\
\hline chess & 36 & - & 36 & 2 & 3196 & - \\
\hline cleve & 13 & 6 & 7 & 2 & 303 & - \\
\hline corral & 6 & - & 6 & 2 & 128 & - \\
\hline $\operatorname{crx}$ & 15 & 6 & 9 & 2 & 692 & - \\
\hline flare & 10 & 2 & 8 & 2 & 1066 & - \\
\hline german & 20 & 7 & 13 & 2 & 1000 & - \\
\hline glass & 9 & 9 & - & 7 & 214 & - \\
\hline glass2 & 9 & 9 & - & 2 & 163 & - \\
\hline hepatitis & 19 & 6 & 13 & 2 & 155 & - \\
\hline iris & 4 & 4 & - & 3 & 150 & - \\
\hline lymphography & 18 & 3 & 15 & 4 & 148 & - \\
\hline monf-3-7-10 & 10 & - & 10 & 2 & 300 & 1024 \\
\hline pima & 8 & 8 & - & 2 & 768 & - \\
\hline satimage & 36 & 36 & - & 6 & 6435 & - \\
\hline segment & 19 & 19 & - & 7 & 2310 & - \\
\hline shuttle-small & 9 & 9 & - & 7 & 5800 & - \\
\hline soybean-large & 35 & - & 35 & 19 & 683 & - \\
\hline vehicle & 18 & 18 & - & 4 & 846 & - \\
\hline vote & 16 & - & 16 & 2 & 435 & - \\
\hline waveform-21 & 21 & 21 & & 3 & 300 & 4700 \\
\hline
\end{tabular}

tools [11]. This discretization method is described by Ting in [20] that is a global variant of the method of Fayyad and Irani [4].

Nineteen of the datasets has no division between training and testing sets. On these datasets the results are obtained by a leave-one-out method inside of the heuristic optimization loop.

On the other hand, two out these twenty one datasets include separated training and testing sets. For these cases, the heuristic optimization algorithm uses only the training set to tune the classifier. A leave-one-out validation is perfomed internally inside of the optimization loop, in order to find the best classifier. Once the best candidate is selected, it is validated using the testing set. The overall process is shown on figure 1 .

\subsection{Results}

Experiments were run in a Athlon $1700+$ with $256 \mathrm{MB}$ of RAM memory. The parameters used to run EDAs were: population size 500 individuals, selected individuals for learning 500, new individuals on each generation 1000, learning type UMDA [16] and elitism. Experiments were run 10 times with two different percentiles: percentile 0.95 $\left(z_{\alpha}=1.96\right)$ and percentile $0.98\left(z_{\alpha}=3.08\right)$.

Results for the UCI problems are shown in the table 2. Results are splitted in two sections, one for the 0.95 percentile $\left(z_{\alpha}=1.96\right)$ and the other for the 0.98 percentile 
Fig. 1. Validacin de los clasificadores cuando el conjunto de datos est dividido en conjunto de aprendizaje y conjunto de testeo.

$\left(z_{\alpha}=3.08\right)$. Columns in the table are: first, the best value obtained with EDAs, second, the average value of the ten executions, third, the average time -in minutes- for the executions and fourth, the improvement respect to a simple naïve Bayes.

Results are really interesting. Respect to naïve Bayes, using the 0.95 percentile, we obtained an average improvement of $3.57 \%$, and using the 0.98 percentile the improvement scales up to $4.65 \%$. It is possible to extract other conclusions from the results. We can appreciate a clear tendency to improve using the 0.98 percentile. Besides, although this is not always true, better improvements are obtained in the data sets with less number of cases.

\section{Conclusion and further work}

In this work we have presented a new semi naïve Bayes approach. In our experiments this approach has an average improvement of $4.65 \%$ respect to the simple naïve Bayes.

As this is the first time we use this approach, many issues remain for future research. For instance, interval estimation naïve Bayes can also be used with continuous variables. It is possible to estimate intervals for the parameters $\mu$ and $\sigma$ of the gaussian distribution.

Also, it is possible to change the objective of the heuristic search. We can try to maximize the area under the ROC curve and not the percentage of successful predictions. Another viable idea is to combine interval estimation naïve bayes with a feature subset selection. On a first phase it is possible to make a subset selection, and on a second phase to apply interval estimation to the previous results.

\section{References}

1. P. Domingos and M. Pazzani. Beyond independence: conditions for the optimality of the simple bayesian classifier. In Proceedings of the 13th International Conference on Machine Learning, pages 105-112, 1996. 
Table 2. Experiment results for Interval Estimation naïve Bayes

\begin{tabular}{|c|c|c|c|c|c|c|c|c|}
\hline \multicolumn{9}{|c|}{ Interval Estimation nä̈ve Bayes } \\
\hline & \multicolumn{4}{|c|}{ Percentile 0.95} & \multicolumn{4}{|c|}{ Percentile 0.98} \\
\hline & $\operatorname{Max}$ & Aver & Time & Improve & $\operatorname{Max}$ & Aver & Time & Improve \\
\hline australian & 88.99 & 88.76 & 10.4 & 3.77 & 90.15 & 89.91 & 11.9 & 4.93 \\
\hline breast & 97.71 & 97.71 & 1 & 0.57 & 97.71 & 97.71 & 1 & 0.57 \\
\hline chess & 92.02 & 91.85 & 107 & 8.19 & 93.74 & 93.70 & 119.4 & 9.91 \\
\hline cleve & 86.14 & 85.91 & 2.5 & 2.31 & 86.47 & 86.47 & 1 & 2.64 \\
\hline corral & 92.18 & 92.03 & 1 & 7.81 & 93.75 & 93.75 & 1 & 9.38 \\
\hline $\operatorname{crx}$ & 89.28 & 89.10 & 13.7 & 2.90 & 90.15 & 89.97 & 10.3 & 3.77 \\
\hline diabetes & 79.82 & 79.51 & 1 & 2.09 & 80.08 & 79.95 & 1.3 & 2.35 \\
\hline flare & 82.18 & 82.18 & 1 & 1.32 & 82.65 & 82.48 & 2.5 & 1.79 \\
\hline german & 80.80 & 80.70 & 23 & 5.40 & 81.80 & 81.60 & 41.2 & 6.40 \\
\hline glass & 79.91 & 79.49 & 1 & 5.14 & 81.31 & 81.31 & 1 & 6.54 \\
\hline glass2 & 88.34 & 88.34 & 1 & 6.13 & 88.34 & 88.34 & 1 & 6.13 \\
\hline heart & 85.93 & 85.89 & 1 & 2.23 & 86.30 & 86.30 & 1 & 2.60 \\
\hline hepatitis & 90.97 & 90.97 & 1 & 5.81 & 90.97 & 90.97 & 1 & 5.81 \\
\hline iris & 96.00 & 95.93 & 1 & 1.33 & 96.00 & 96.00 & 1 & 1.33 \\
\hline letter & 75.08 & 74.97 & 301 & 1.04 & 75.60 & 75.37 & 340 & 1.56 \\
\hline lymphography & 92.57 & 92.09 & 1 & 7.43 & 95.27 & 94.66 & 2.6 & 10.13 \\
\hline monf-3-7-10 & 96.45 & 95.85 & 2 & 12.24 & 100.00 & 100.00 & 2 & 15.79 \\
\hline pima & 79.69 & 79.47 & 1 & 1.96 & 80.08 & 79.90 & 1 & 2.35 \\
\hline satimage & 83.33 & 83.23 & 221.1 & 0.87 & 83.42 & 83.42 & 228.4 & 0.96 \\
\hline segment & 93.55 & 93.53 & 131.1 & 1.60 & 95.45 & 95.41 & 155.4 & 3.50 \\
\hline shuttle-small & 99.43 & 99.43 & 20.6 & 0.07 & 99.90 & 99.88 & 22.5 & 0.54 \\
\hline soybean-large & 94.00 & 94.00 & 180.9 & 0.88 & 94.58 & 94.58 & 188.5 & 1.46 \\
\hline vehicle & 66.78 & 66.56 & 19.2 & 5.31 & 70.33 & 70.20 & 26.5 & 8.86 \\
\hline vote & 91.49 & 91.47 & 1 & 1.38 & 95.17 & 95.17 & 2 & 5.06 \\
\hline waveform-21 & 83.08 & 83.03 & 133.8 & 1.44 & 83.60 & 83.52 & 162.3 & 1.96 \\
\hline
\end{tabular}

2. J. Dougherty, R. Kohavi, and M. Sahami. Supervised and unsupervised discretization of continuous features. In International Conference on Machine Learning, pages 194-202, 1995.

3. R. Duda and P. Hart. Pattern Classification and Scene Analysis. John Wiley and Sons, 1973.

4. U. Fayyad and K. Irani. Multi-interval discretization of continuous-valued attributes for classification learning. In Proceedings of the 13th International Conference on Artificial Intelligence, pages 1022-1027, 1993.

5. N. Friedman, D. Geiger, and D.M. Goldszmidt. Bayesian network classifiers. Machine Learning, 29(2-3):131-163, 1997.

6. J. Gama. Iterative bayes. Intelligent Data Analysis, 4:475-488, 2000.

7. I.J. Good. The Estimation of Probabilities: An Essay on Modern Bayesian Methods. MIT Press, 1965.

8. D.J. Hand and K. Yu. Idiot's Bayes - not so stupid after all? International Statistical Review, 69(3):385-398, 2001.

9. J. Kohavi, B. Becker, and D. Sommerfield. Improving simple Bayes. Technical report, Data Mining and Visualization Group, Silicon Graphics, 1997. 
10. R. Kohavi. Scaling up the accuracy of naïve-Bayes classifiers: a decision-tree hybrid. In Proceedings of the Second International Conference on Knowledge Discovery and Data Mining, pages 202-207, 1996.

11. R. Kohavi, G. John, R. Long, D. Manley, and K.Pfleger. Mlc++: A machine learning library in c++. Tools with Artificial Intelligence. IEEE Computer Society Press, pages 740-743, 1994.

12. I. Kononenko. Semi-naive bayesian classifier. In Sixth European Working Session on Learning, pages 206-219, 1991.

13. P. Langley. Induction of recursive Bayesian classifiers. In European Conference on Machine Learning. Berlin: Springer-Verlag, pages 153-164, 1993.

14. P. Langley and S. Sage. Induction of selective Bayesian classifiers. pages 399-406, 1994.

15. P. Larrañaga, J.A. Lozano, and E. Bengoetxea. Estimation of Distribution Algorithms based on multivariate normal and Gaussian networks. Technical Report KZZA-IK-1-01, Department of Computer Science and Artificial Intelligence, University of the Basque Country, 2001.

16. H. Mühlenbein. The equation for response to selection and its use for prediction. Evolutionary Computation, 5:303-346, 1998.

17. P. M. Murphy and D. W. Aha. UCI repository of machine learning databases. http://www.ics.uci.edu/ mlearn/, 1995.

18. M. Pazzani. Searching for dependencies in Bayesian classifiers. In Proceedings of the Fifth International Workshop on Artificial Intelligence, pages 239-248.

19. M. Ramoni and P. Sebastiani. Robust learning with missing data. Machine Learning, 45(2):147-170, 2001.

20. K.M. Ting. Discretization of continuous-valued attributes and instance-based learning. Technical Report 491, University of Sydney.

21. G.I. Webb and M.J. Pazzani. Adjusted probability naïve bayesian induction. In Australian Joint Conference on Artificial Intelligence, pages 285-295, 1998.

22. M. Zaffalon. A credal approach to naive classification. In Proceedings of the 1st International Sysmposium on Imprecise Probabilities and Their Application, pages 405-414, 1999.

23. M. Zaffalon, K. Wesnes, and O. Petrini. Credal classification for dementia screening. Artificial Intelligenci in Medicine, LNAI 2101, pages 67-76, 2001.

24. W. Zhou and R. Greiner. Learning accurate belief nets, 1999. 
Fig. 2. Interval Estimation naïve Bayes. 